Revista Digital Año 4. No 4 - Año 2013. --pág. 1 - 62

ISSN 1853-1393

Resistencia. Chaco. Argentina - 2013

\title{
La enseñanza de la Historia en el nivel medio y la formación del profesor en Historia
}

\section{The teaching of History in the middle and teacher formation in History}

\author{
Irma Esther Rosso
}

Fecha de recepción: 18-03-2013

Fecha de aceptación y versión final: 20-05-2013

Resumen: Los motivos que originaron esta proyecto de investigación, se basan en las observaciones que realizan los alumnos de la cátedra Didáctica Específica y Pasantías en Historia de la Facultad de Humanidades de la UNNE, en las instituciones del nivel medio y terciario; las reflexiones que aportan sobre la realidad que observan, la relacionan con la formación que han recibido en la universidad.

A través de los diarios de itinerancia, que confeccionan los alumnos-pasantes (o alumnos-docentes), el análisis de las planificaciones de los docentes y las entrevistas, cada año aportan nuevos elementos que les permiten caracterizar (conocer) la realidad de la enseñanza de la Historia en las Instituciones de nivel secundario; a su vez reflexionan sobre su propia formación docente en proyección hacia la práctica profesional en el período de pasantía.

Reflexiones, que al momento de hacerse cargo de los cursos y comenzar las prácticas docentes, se convierten en cuestiones problemáticas que generan miedos, consultas permanentes y el reconocimiento de no haber "aprendido" a resolver problemas prácticos en las aulas.

De esta manera comienzan los planteos sobre la "formación docente", reconocen que es muy teórica, que manejan muy bien los contenidos disciplinares, no así las teorías pedagógicas, pero sobre todo llevarlas a la práctica. En este artículo se presentan las conclusiones que se generaron durante el período 20122013.

Palabras claves: Didáctica de la Historia - Formación Docente - Teoría/práctica.

Abstract: The reasons that led to this research project is based on observations made by / the alumni / ae of the chair Specifies Teaching and Internships in History, Faculty of Humanities UNNE, institutions for secondary and tertiary, the reflections that bring about the reality they observe, relate to the training received in college.

Through roaming newspapers, who make the student- interns (or student- teachers), analysis of schedules and interviews teachers, each year they bring new elements to characterize ( know ) the reality of teaching of History in secondary level institutions, in turn reflect on their own teacher training outreach to professional practice internship period.

Reflections, which at the time of taking over the courses and begin teaching practices become problematic issues that generate fears, ongoing consultation and recognition of not having "learned" to solve practical problems in the classroom.

They begin the proposals on "teacher training", recognize that it is very theoretical, they handle very well the disciplinary content, pedagogical theories and no, but mostly implement them. This article presents the findings that were generated during the period 2012-2013

Key words: Teaching History-Teacher Education- Theoryand practice.

\footnotetext{
${ }^{1}$ Facultad de Humanidades - UNNE - correo electrónico: rossocuqui@ gmail.com
} 
La enseñanza de la Historia en el nivel medio es un desafío para los docentes, porque con su pretendida objetividad científica (Fontana, Josep, 2001:181) fue integrada a los diseños curriculares como "la materia" que tenía una utilidad ejemplificadora para formar ciudadanos y podía justificar la política implementada por los diferentes gobiernos. Este modelo de disciplina a enseñar en el nivel secundario (o denominado también nivel medio) sigue vigente, en la actualidad.

Para lograr esos objetivos, la Historia debe presentar modelos a seguir como buen ciudadano. Perspectiva que la marcó como "la materia" que explicaba los hechos relevantes (prototipos), narraba la cadena de hechos ejemplificadores que debían ser "internalizados" por los estudiantes, sin necesidad de comprenderlos sí, de practicarlos; de esta manera, la Historia pasó a ser sinónimo de memorización mecánica de fechas, hechos y personajes en que prevalecían acontecimientos políticos-militares. Con estas características se ha enraizado profundamente en los planes de estudio y en las prácticas de enseñanza que, aun hoy, perviven, en nuestro país. De acuerdo con esta perspectiva, la historiografía se basaba en el uso de textos narrativos con datos encadenados cronológicamente, sin una relación reticular de los mismos (corriente metódica) y el rol docente se limitaba a presentarlos como tal y verificar (evaluar?) si los alumnos habían logrado memorizarlos. Es decir, existe coincidencia entre una historia "objetiva" fundada en fechas y datos y una didáctica bancaria en que el docente presenta los contenidos y los alumnos, todos por igual lo memorizan.

El proceso de investigación histórica, a partir del siglo XX, fue girando su centro de atención y de análisis hacia todos los aspectos que hacen a la vida de una sociedad, transformándose en la ciencia que analiza los procesos de cambio (coyunturas) y estructuras por los que atraviesa la sociedad, observando las constantes, los cambios en el tiempo, estudia la multicausalidad reticular de los acontecimientos, se propone indagar en el sentir y accionar, no sólo de los personajes destacados, sino especialmente de los sujetos anónimos que viven y conforman la sociedad, tratando de interpretar los sucesos y comprender al ser humano, inserto en su realidad histórico social.

Estos cambios que sufre la Investigación histórica, en el estudio de su campo categorial (Moradiellos, 2000) está cuestionando las formas de enseñar (Rockwell, 2000:145) la disciplina, lo que lleva a un cambio en la formación que reciben los futuros profesores en Historia. Porque ante la perspectiva de interpretar y de comprender a las sociedades en el pasado, se amplían las fuentes y documentos históricos (no son sólo se consideran los escritos oficiales, sino que se tienen en cuenta documentos privados, escritos, orales, materiales culturales, monumentos, elementos de la vida cotidiana, que permiten caracterizar los modos de vida), con los que trabaja el investigador y están al alcance del docente; de esta manera tiene acceso a documentación y huellas dejadas por la humanidad, que le permite, al docente, analizarla junto con los alumnos para llegar a la comprensión de la realidad histórico social de una determinada época; situación que concuerda con la perspectiva didáctica del constructivismo, en que docente y alumnos construyen el conocimiento.

Sin embargo, estos cambios no se observan en la práctica de la escuela secundaria; la perspectiva historiográfica y la didáctica de la Historia atraviesan serios planteos, por falta de actualización. Los alumnos pasantes, de la asignatura Didáctica Específica y Pasantía en Historia, ante esta realidad, consultan a los docentes en ejercicio, quienes responden que se debe a la formación que han recibido. Motivos que condujeron a esta investigación, cuyo objetivo específico era identificar los fundamentos epistemológicos de la disciplina que manejan los docentes y la forma de enseñarlos -didáctica de la Historia-, en el nivel medio (o escuela secundaria) y la 
formación para el ejercicio de la profesión; además, analizar la vivencia de su formación en los alumnos pasantes, del último año de la carrera del profesorado en Historia, cursantes de la asignatura antes mencionada.

La Formación Docente en el ámbito universitario, en este momento está seriamente cuestionada, porque existe un desfasaje entre lo que se considera la vieja y la nueva historia. Es decir, se opta por un enfoque actual de la disciplina pero, se continúa exigiendo su memorización; aduciendo "postura de la cátedra"; "los autores reconocidos consideran este acontecimiento", "este hecho se interpreta de esta manera", con estos fundamentos se orientan al estudiante en una sola perspectiva de análisis, elegida por el docente no dando opción a analizar otras corrientes historiográficas.

En este artículo se presentan las primeras conclusiones de un trabajo de investigación sobre "La enseñanza de la Historia en el nivel medio y la formación del profesor en Historia desde la perspectiva del alumno-pasante", llevado a cabo por los alumnos/pasantes y el equipo docente de la cátedra Didáctica Específica en Historia y Pasantía, de la Facultad de Humanidades de la Universidad Nacional del Nordeste.

La cátedra, propicia un espacio de autoformación, en que los alumnos-pasantes tomaron como anclaje de análisis: la práctica de la enseñanza y la formación docente que han recibido, para la comprensión de su andamiaje y dinámica; en ese proceso de análisis aplican las técnicas de investigación educativa a su propio accionar en la práctica profesional, durante el período de pasantías docentes, a través de a.autobiografías del estudiante universitario b.- la confección de un diario de itinerancia durante el período de pasantías, que les permite la autorreflexión y realizar valiosos aportes sobre la formación docente que brinda la institución universitaria; c.observación de clases y d.- análisis de planificaciones de los cursos que observan, en el nivel medio (o escuela secundaria).

Utilizaron el método histórico-crítico y hermenéutico, lo que les permitió analizar la realidad que se vive en las aulas, para interpretar el accionar de los sujetos y cotejar con su formación, en relación con las diferentes teorías pedagógicas y emitir juicio crítico del tránsito por las aulas universitarias.

Es histórico-crítico porque este proceso de trabajo sistemático, la cátedra lo implementa desde el año 2001; pero, desde el año 2006 lleva un registro de las actividades que se desarrollan. De las conclusiones anuales, que cada semestre se retoman, analizan y comparan actualizándolas, se ha ido confirmando el diagnóstico inicial: la enseñanza de la Historia es tradicionalista, en los establecimientos secundarios, sin embargo existentes docentes que favorecen una práctica ecléctica en todo sentido, desde la perspectiva historiográfica hasta el estilo didáctico. Los datos relevados, por medio de un análisis hermenéutico, describen la situación de clase y el sentir de los protagonistas, permitiendo la compresión de la realidad escolar y universitaria.

Estas actividades requieren una participación activa del alumno-docente (o alumnopasante), junto al docente tutor -del nivel medio o terciario y del equipo de cátedra-; con el objeto de observar la situación escolar para comprenderla tal cual la perciben los sujetos de acuerdo con sus experiencias y sentimientos (Carr y Kemmis, 1988).

Las tareas son desarrolladas en dos fases. La primera, se inicia con entrevista a los docentes en ejercicio, la observación de clases, en establecimientos de nivel medio (o secundario) en escuelas de zonas periféricas (3-tres) y del micro centro (3-tres-), ${ }^{i}$ analizando específicamente la práctica de la enseñanza de la disciplina. 
La segunda fase consiste en el análisis de las tareas preactivas de los docentes, en que se toman en consideración las planificacionesii; de los profesores de los cursos que observan y en forma conjunta -alumnos/pasantes y docentes tutores-, establecen la triangulación entre el enfoque de la disciplina, es decir las corrientes historiográficas que implementan los docentes, cómo conciben la disciplina, los estilos didácticos aplicados, en este caso determinaron la correspondencia entre las corrientes historiográficas y la didáctica de la Historia.

Finalmente los alumnos/pasantes reflexionan sobre su futura práctica profesional en el marco de la formación docente que han recibido, a lo largo de cinco años.

Los resultados de estas tareas, se basan en el estudio de los datos mencionados, de acuerdo con las tres categorías de análisis propuestas por Shulman (1989):

1.- El conocimiento del contenido (o de la materia) es el conocimiento de la disciplina, que se deriva de la ciencia, lo que comprende el especialista (profesor de Historia) sobre su especialidad, pero también hace referencia al conocimiento actualizado, de las diferentes posturas o escuelas históricas en relación directa con las normas usadas en la construcción del saber científico.iii

2.- Conocimiento pedagógico (o práctico profesional) es la comprensión de los problemas $\mathrm{y} / \mathrm{o}$ dificultades que presentan ciertos temas para su análisis y aprendizaje, este conocimiento incluye las conceptualizaciones y la terminología; los marcos conceptuales de los diferentes paradigmas y los conceptos puentes (en relación con otras disciplinas) ${ }^{\text {iv }}$. Según el dominio, que el docente, tenga de estos conocimientos, decidirá el uso de documentos y fuentes históricas, libros de textos, entre otros materiales.

3.- Conocimiento curricular (experiencia o saber hacer en la práctica) es el conocimiento sobre la selección y organización del conocimiento a enseñar que se refiere, específicamente a qué y cómo enseña la disciplina el profesor.

De acuerdo con estas categorías, se han analizado las entrevistas, las observaciones de clases y las planificaciones, los resultados se triangulan para obtener la conclusión.

Posteriormente, se coteja con los diarios de itinerancia y las autobiografías de los estudiantes universitarios, para determinar la correspondencia entre la formación que reciben y la realidad en la que deben ejercer la profesión docente.

\section{Análisis de los datos}

Se presentan los datos analizados, de acuerdo con las categorías mencionadas:

1.- Conocimiento de la materia: en este caso, en la entrevista, se realizó una pregunta estructurada pero sin ninguna alternativa de respuesta, en que se solicitó definir el concepto de Historia, en las observaciones de clases se analizaron las explicaciones de los docentes y cómo se enunciaban los contenidos en las planificaciones

2.- Conocimiento pedagógico: en la entrevista se solicitó cómo define -el docenteel concepto Historia para sus alumnos, en las observaciones, como aborda el tiempo histórico y su división, finalmente cómo se halla estructurado en las planificaciones

3.- Conocimiento curricular: se preguntó qué criterios usa, el docente, para organizar los contenidos, cómo los relaciona con otras ciencias humanas, establece conceptos puentes, cómo los enseña, cuál es su rol docente y que espera de los 
alumnos, de igual manera se trató de identificar estos elementos en las observaciones de clases y planificaciones

En el análisis de las respuestas, se ofrece primero la presentación cuantitativa, para abordar luego, el análisis cualitativo.

El cuestionario cuenta con preguntas estructuradas y abiertas en las que se solicita la fundamentación de las respuestas.

\section{Entrevistas}

I.- Conocimiento de la materia: Concepto de la disciplina Historia y la concepción del tiempo

II.- Conocimiento pedagógico: organización de los contenidos, su relación con otras disciplinas y enseñanza.

La primera pregunta, todos los docentes la pasaron por alto porque manifestaron que, la misma concepción que manejaban de Historia era la que enseñaban a sus alumnos. Motivo por el cual, se analizó el conocimiento de la materia y el conocimiento pedagógico en forma conjunta. Aclarada esta situación, se presenta el análisis.

Las primeras preguntas de la encuesta, hacen referencia a dos conceptos que los profesores en Historia deberían manejar, si no uniformemente, tendría que existir una aproximación certera en su uso y concepción porque, solicitan la definición del campo categorial de estudio de la disciplina ${ }^{v}$ de la cual son especialistas, en un caso y en el segundo caso debían responder sobre la secuenciación del tiempo, para observar el grado de actualización al respecto y la coincidencia (o no) con el conjunto de las respuestas dadas.

\section{Pregunta.- Cómo define, para sus alumnos, el concepto de Historia}

El $30 \%$ de los docentes respondió que la define como ciencia social, el $20 \%$ es una ciencia; el $15 \%$ estudia hechos del pasado; $10 \%$ estudia el pasado del hombre/ pasado humano; $8.7 \%$ estudia los hechos trascendentes o destacados; las respuestas continúan en un porcentaje que oscila entre el 4 , el 2 y el $1 \%$ estableciendo, las siguientes respuestas: -Conocimiento sistemático del pasado, -Estudia los hechos significantes e importantes, -Ciencia que posee objeto y método de estudio, -Estudia pasado, presente y futuro, -Es para comprender la realidad, -Enseña a pensar y a criticar, -Sirve para formar el sentimiento de patria, -Ciencia activa en construcción según las causas y efectos que producen los hechos históricos relevantes, -Es el conjunto de acciones del hombre, -Tiene utilidad para comprender el presente, Estudio del hombre y sus acciones pasadas, -Conoce el pasado con ayuda de las ciencias auxiliares.

A partir de la lectura y análisis de las respuestas se observa la disparidad de opiniones, incluso de interpretación de la consigna, en que confunden el concepto de Historia como Ciencia (rerumgestarum) y la Historia como acontecer de la vida humana (res gestae).

A pesar de que la pregunta era precisa, se debía brindar una definición del término Historia como ciencia, algunos establecen sus objetivos: Enseñar a pensar y a criticar o bien, "sirve para formar el sentimiento de patria. En este punto observamos "la falta 
de interpretación de la consigna"; o bien la confusión de los docentes al tratar de definir un concepto tan bien estudiado y aprendidos por ellos.

Con respecto a la segunda pregunta, referida a la organización y secuenciación del tiempo, se solicitaba:

\section{Pregunta.- Como estructura la secuencia temporal}

El 59\% de los profesores respondieron -"según la periodización tradicional", el $52 \%$-"según procesos"; algunos docentes aclararon que usan una -"secuencia temporal hechológica"; otros se inclinan por -"la que traen los libros de texto"; en tanto que algunos docentes reconocieron que trabajan con la "tradicional para la historia universal y en procesos para Historia Argentina"

No hay precisión en las cifras debido a que, algunos docentes eligieron más de una opción, estableciendo que varían la organización del tiempo, de acuerdo con los temas o bien, si enseñan historia universal o historia argentina.

A partir del análisis cuantitativo de las respuestas observamos que aproximadamente un $60 \%$ de los docentes continúan aplicando la periodización tradicional para explicar la Historia; en tanto que un $52 \%$ establece que usa la perspectiva de procesos, es decir que aplican los lineamientos de Ferdinand Braudel (1992), con respecto a la duración de los procesos -en el tiempo- que se los puede ubicar en momentos de larga, media y corta duración.

En la fundamentación algunas respuestas hacen referencia al uso de la periodización tradicional porque:

a.- "es la única que conocemos, porque así lo aprendimos en la carrera de formación de profesores de historia"

b.- "es la que manejan los alumnos y más conocida"

c.- "es la más conocida y usada en el nivel medio"

d.- "es la que mejor ubica a los alumnos"

e.- "la usamos para estudiar Historia universal o eurocentrista" (sic)

f.- "es la única que ofrece conocimientos precisos"

g.- "es la que habitualmente traen los libros"

En tanto que el uso de una organización diacrónica que demanda el enfoque por procesos, reconocen que se:

a.- "se comprende mejor"

b.- "la usamos para enseñar Historia Argentina, en tanto la periodización tradicional la aplico para explicar historia universal"

c.- "se observa mejor cuáles fueron los cambios y permanencias"

Es decir, que no existe uniformidad de criterios sobre organización de la secuenciación temporal. Se observa una situación engorrosa cuando utilizan, dos categorías diferentes de periodización, la tradicional para enseñar historia universal y una procesual para desarrollar historia argentina, no quedando explicito por qué este cambio, como así tampoco si los alumnos comprenden las maneras diferentes de 
organizar el tiempo y las relaciones que deben existir de la política argentina en el marco internacional.

No se observa un cuestionamiento ni proposiciones nuevas, para abordar el análisis del tiempo histórico; de lo que se deduce un desconocimiento del pensamiento de Koselleck, ${ }^{\mathrm{vi}}$ autor que realiza propuestas de análisis desde una relación entre la historia conceptual, historia social y semántica histórica, acorde con el constructivismo (estilo didáctico a la que la mayoría de los docentes se adhieren, como veremos más adelante)

De acuerdo con las preguntas anteriores se formuló una pregunta, encaminada a observar el manejo de las actuales corrientes historiográficas, si desarrollan los contenidos desde una sola perspectiva histórica 0 , usando diferentes autores, permiten a los alumnos conocer el análisis desde distintas escuelas históricas 0 ideologías. Los resultados fueron:

Pregunta.- Ofrece a sus alumnos, la posibilidad de analizar los temas desde: a.diferentes autores, b.-diferentes corrientes historiográficas, c.-un sólo autor y d.-sólo lo que el docente interpreta (se ofrecieron cuatro opciones, que debían fundamentar la elección)

La mayoría de los profesores, un $84 \%$, respondieron que se manejan con "diferentes autores", en tanto que el $12 \%$ trabaja con "diferentes corrientes historiográficas", el $7.1 \%$, usa "un solo autor" y el $1.7 \%$ estableció "sólo lo que el docente interpreta".

Se observa que el $84 \%$ de los profesores permite el trabajo con diferentes autores, es decir que se supone que enseñarían desde una didáctica hermenéutica, permitiendo a los estudiantes indagar en las diferentes corrientes historiográficas; sin embargo, al relacionar esta respuesta con la pregunta sobre la periodización la mayoría respondió que trabaja con la tradicional que admite solo un desarrollo lineal y preciso de los hechos a través de mención de causas-consecuencia, es decir, no hay correlación entre la lectura de varios autores y un punto de vista positivista de la periodización. Esta apreciación se corresponde con la fundamentación que aportan los profesores, cuando aclaran que son diversos los autores, porque utilizan los textos que posee la biblioteca escolar, con libros antiguos (de las décadas de 1940-50) desde Ibáñez y Astolfi hasta los más recientes enviados por el Ministerio Nacional, que readaptaron sus índices a lo requerido en el diseño curricular, sin que exista realmente un cambio en la perspectiva de análisis de los acontecimiento históricos, de acuerdo con las nuevas corrientes de Annales o historia conceptual, es más, la mayoría de los textos actuales se inician con una línea de tiempo según la periodización tradicional.

Otras fundamentaciones, afirman: "rechazo trabajar con las diferentes corrientes historiográficas, porque opino que es muy difícil para alumnos del nivel medio", en otros casos el problema gira en torno a la formación que tuvieron "no estamos preparados porque en mi formación como profesora de Historia no vi (sic) las distintas escuelas historiográficas, solo memorizamos los contenidos", en tanto que $7.1 . \%$ fundamenta que trabajan con un solo autor porque es "más cómodo y evita tener que trabajar con fotocopias".

\section{III.- Conocimiento curricular: criterios que usa para organizar los contenidos.}

En este bloque de preguntas, referidas a la confección de la planificación, se solicitaba en primera instancia la explicitación sobre la selección y organización de los 
contenidos, aplicación de los principios de interdisciplinariedad en el desarrollo de las clases, al relacionar conceptos o temas históricos con conocimientos de otras disciplinas.

La pregunta sobre la organización de los contenidos temáticos, arrojaron los siguientes resultados:

Pregunta.- Qué criterios utiliza para organizar los contenidos de la planificación anual

El 55\% de los profesores respondieron que organiza "por ejes temáticos", el $41 \%$ establecieron "respeto el Diseño curricular", un 32\% "lo organizo por unidades", un $25 \%$ contestó "los organizo teniendo en cuenta las características psicológicas de los alumnos" $12.5 \%$ "los organizo en función de los textos que recomiendo a los alumnos" y un $8.9 \%$ "organizo alrededor de un concepto"

Los porcentajes demuestran que la mayoría de los docentes respetan el Diseño curricular, organizando la planificación por ejes temáticos. En la fundamentación establecen la necesidad de respetar la legislación vigente, por las visitas de autoridades (supervisores) que "controlan el desarrollo de las planificaciones."

Otros docentes responden que unen temas y unidades, reconociendo que no saben la diferencia entre dividir por temas y por unidades.

Es considerable el número de docentes que opta por respetar las características psicológicas de los alumnos. En la fundamentación explican que respetan "los intereses de los alumnos al momento de desarrollar la clase", es decir, observamos una confusión entre características psicológicas de los alumnos para realizar la trasposición didáctica y los intereses de los alumnos.

Lo llamativo es que sólo de $8.9 \%$ de profesores organiza la planificación, según conceptos.

Es necesario dejar explícitamente aclarado que, los porcentajes enunciados en los párrafos anteriores, reflejan la opinión de los docentes en la entrevista, sin contar con la planificación a la vista, para corroborar sus respuestas.

La pregunta referida específicamente a la didáctica de la Historia, requería:

Cómo enseña Historia (se ofrecieron cuatro opciones, con la debida fundamentación y una quinta que se podía agregar libremente)

\begin{tabular}{|l|l|}
\hline a.- Exponiendo (explicitando) los temas & $42 \%$ \\
\hline b.- Expositivo con participación de los alumnos & $75 \%$ \\
\hline c.- Los alumnos exponen y Ud. realiza las aclaraciones pertinentes & $35 \%$ \\
\hline d.- Los alumnos preparan clases especiales en grupos & $35 \%$ \\
\hline e.- Otros. Indique & $12.5 \%$ \\
\hline
\end{tabular}


La mayoría de los docentes desarrollan sus clases, de manera "magistral" porque respondieron que exponen los temas, pero permiten la participación de los alumnos.

La opción e.- la han elegido un 12.5\% de docente pero, ninguno explicitó en qué consistía su estilo didáctico

Es llamativo la cantidad de docentes que defienden la "preparación de clases especiales" y la exposición de lecciones, como la mejor forma de evaluar a los estudiantes y en la fundamentación establecen que con esta estrategia didáctica "los entrenan" para hablar en público y califican la expresión oral.

\section{Conclusión sobre las entrevistas a docentes}

Del análisis de las entrevistas realizadas a treinta docentes, los alumnos/pasantes y los profesores tutores con el equipo de cátedra, infieren que, desde el punto de vista del conocimiento de la materia y del conocimiento pedagógico:

no existe una concepción de la disciplina que se pueda decir que se aproximan, de manera unívoca, en el uso del concepto del término Historia,

$>\quad$ manifiestan concepciones erróneas y/o desactualizadas

$>\quad$ confusión entre definir un concepto y su utilidad

$>\quad$ se continua con una historia encaminada a la memorización antes que a la comprensión de realidad histórico social

se continua con la aplicación de la organización temporal tradicional, es decir una historia hechológica, en que se priorizan las fechas (preceptiva metodológica)

Desde el punto de vista del conocimiento curricular, se ha detectado que:

no existe como marco de referencia las corrientes historiográficas en vigencia, que sean el sustento de criterios selección y organización de contenidos

$>\quad$ los docentes manifiestan en su mayoría que exponen los temas, permiten preguntas a los alumnos para aclarar temas, no para generar debates, se evidencia que los profesores son mediadores en los significados concretos que obtienen los alumnos ${ }^{\text {vii }}$

$>\quad$ los docentes se consideran "guías" del aprendizaje de sus alumnos, pero realizando preguntas cerradas, con opciones a una sola respuesta

\section{Planificaciones y observaciones de clases}

De los docentes entrevistados, cinco manifestaron predisposición inmediata para que se pudieran realizar las observaciones, en sus cursos. En primer lugar se les solicitó las planificaciones y en una segunda entrevista, realizaron las aclaraciones pertinentes sobre la confección de las mismas.

El análisis de las planificaciones, se corresponde con los criterios utilizados para las entrevistas, porque al buscarse la correspondencia entre el conocimiento del docente y su aplicación se continuó con las mismas categorías de análisis. En líneas generales, 
los docentes reconocieron que hay acuerdo en el Departamento de Ciencias Sociales de las instituciones escolares para respetar los diseños curriculares del ministerio público, sin modificaciones. Se detecta cambios sólo, en la elaboración de los ítems que conforman una planificación como por ejemplo el enunciado de: objetivos, actividades, procedimientos.

\section{Se presentan a continuación el análisis de las planificaciones y clases observadas.}

La docente Carmen, manifestó que su planificación se corresponde con el currículo vigente, es más es una copia del mismo

En tanto que la profesora María Clara, que es docente del mismo establecimiento, su planificación, cuenta con expectativas de logros, los contenidos son temáticos/ conceptuales, si bien enuncia contenidos procedimentales, están redactados en términos de actividades y evaluación, no presenta bibliografía ni cronograma. La docente comenta que fue la manera en que aprendió a confeccionar la planificación; sin embargo los contenidos al igual que la docente anterior es copia del diseño curricular vigente

Alba, profesora de segundo año, explicó que la planificación responde a un "programa" en el que establecen los temas a desarrollar divididos en unidades, carecen de enunciados, de fundamentación, objetivos o propósitos, criterios de evaluación, en la entrevista personal la docente alega que son directivas de la institución que la establecen al inicio del año escolar.

El profesor José Luis explica que elaboró la planificación según su propio criterio, manifestó que no tiene directivas específicas de las autoridades de la institución, por lo tanto "respeté la planificación "dejada por (apellido del docente) que se jubiló y yo seguí aplicando la misma"

En el caso de Mercedes, aclaró que se respetan las directivas de la rectora del establecimiento, quien determina qué ítems deben figurar en las planificaciones, es decir deben constar los objetivos generales, objetivos específicos, contenidos temáticos, actividades de los alumnos, actividades del docente, evaluación (se enunciaban instrumentos de evaluación no criterios), bibliografía del alumno, bibliografía del docente y cronograma. En la entrevista la docente aclaró que las planificaciones son "controladas" por la jefa del departamento correspondiente y celosamente archivadas; con respecto a los contenidos siguen los lineamientos del diseño curricular de la provincia.

En líneas generales observamos la disparidad de "modelos" de planificaciones, sin embargo en cuanto a los contenidos todas respetan las directivas del ministerio de educación, por lo que resulta un verdadero problemas determinar sobre qué criterios seleccionan y organizan los conocimientos de la materia y los conocimientos pedagógicos, los docentes, porque se limitan a copiar los temas del diseño curricular. Es necesario aclarar que en la jurisdicción-Chaco-, existen diferentes diseños curriculares, porque aún subsisten currículos para escuelas de orientaciones polimodales, escuelas de enseñanza media, escuelas rurales, de jornadas completa; además en el espacio de la institución, en algunos establecimientos se ofrecen las horas a los profesores de historia para enseñar historia regional o provincial, en tanto que en otros, estos temas no se desarrollan e incrementan las horas de matemáticas o lengua. 
Motivos que llevaron a direccionar la investigación hacia las observaciones de clases, para establecer la triangulación con las entrevistas. El acuerdo que se tuvo con los docentes fue observar las clases desde el inicio hasta la finalización de un tema, el cual lo seleccionaría el docente del curso, por lo tanto, no existió una número determinado de clases a observar, se puede decir que fluctuó entre 10 y 14 módulos.

El autor Wittrock (1989) cuando se pregunta ¿qué es la observación? responde, depende del propósito que mueve a la persona que la formula. Afirma que cuando "la observación se usa para responder a una pregunta formulada, debe ser deliberada y sistemática" y llevarla a cabo como un proceso consciente que logre explicarse de modo que otras personas puedan apreciar su adecuación y comprender el trabajo de investigación. De acuerdo con esta concepción, el mismo autor propone una tabla de criterios a aplicar para la adecuación de la observación de Genishi Y Hymes, que se tomaran como modelo en el presente trabajo viii

Los autores indican que un tipo de criterios a tener en cuenta es la concordancia entre el foco de la observación, las preguntas para la investigación, la recolección de datos, teniendo en cuenta el nivel de análisis.

Utilizando la tabla que proponen los autores se diseñó el plan de la siguiente manera:

Las preguntas de la investigación: Observar la concordancia entre las concepciones de la disciplina que tienen los profesores, las planificaciones y la forma de enseñar esas concepciones

Nivel de análisis: descriptivo

Determinar la transcripción: se tratará de establecer las orientaciones historiográficas que transmiten los docentes en sus clases de historia, a través del desarrollo de diferentes temas.

\section{Observaciones}

Se presenta la descripción de las observaciones en relación con las planificaciones de cada docente, por lo tanto se colocan los nombres de cada uno de ellos.

La docente Carmen, aclara que la planificación es un trámite que se cumple, por lo tanto prepara las clases de acuerdo con el nivel de los alumnos de quinto año(es una escuela céntrica, el nivel socio económico de los alumnos que asisten es medio-alto, la mayoría son hijos de profesionales, la escuela está en buenas condiciones edilicias)

El tema es La revolución libertadora, la docente realiza un esquema en el pizarrón, a medida que lo confeccionaba explicaba, respetando las fechas y acontecimiento, los alumnos registran en sus carpetas el esquema, con anotaciones personales.

Desarrollado el tema, los alumnos pasan a trabajar sobre un texto de Ernesto Sábato, que consiste en la lectura y análisis; además, de responder un cuestionario. La tarea la realizan en grupos, por lo que se genera un dialogo entre los grupos y por momentos debates.

La docente recorre los grupos aclara dudas y explica algunos términos desconocidos por los alumnos, al finalizada la lectura deben entregar una síntesis. Trabajo realizado en tres módulos; al término de los cuales se continua con las consecuencias de la revolución repitiéndose el mismo esquema de desarrollo.

En estas clases la docente se limita "enunciar" los acontecimientos ocurridos, no explica la realidad histórico social desde ninguna corriente historiográfica ni ideológica. 
Las clases son un continuo de los "hechos" ocurridos en la década del 50 en nuestro país, centralizado en el acontecer político. Es la docente que en la entrevista explicó que enseñar desde diferentes corrientes historiográficas en difícil para los alumnos, porque no están preparados; por lo tanto "no interesa donde estoy parada", o desde qué perspectiva enseño.

Hay una concepción de Historia academicista, es decir hechológica sin la relación reticular necesaria.

María Clara, es profesora en un quinto año, en la misma institución que ejerce Carmen. El tema a desarrollar es Caída de Frondizi, la docente enuncia el tema y entrega un texto de Abelardo Ramos, los alumnos debían leer y extraer las causas de la caída del presidente; previa explicación de la técnica procedimental lectura de texto histórico. Después de dos módulos, que utilizaron para la lectura y análisis del texto, en el tercer módulo lograron realizar, en forma conjunta con la profesora, un cuadro en el pizarrón. Es decir, que se desarrolló el tema desde la perspectiva de un solo autor. Se continuó con la lectura del mismo autor, desarrollándose la década del 60 en Argentina

Los estudiantes a pesar que entraron en contacto directo con el conocimiento, la profesora sólo enunciaba el tema, realizaron una síntesis, no hubo intercambio de opiniones, ni se les solicitó emitir un juicio crítico. Es decir que se limitaron a aplicar la técnica procedimental, como estaba previsto en la planificación y confeccionar una síntesis, en este sentido hay concordancia entre la planificación y el desarrollo de la clase, no así con los conceptos enunciados, porque fue una síntesis de la opinión de autor del texto.

Alba es docente en un segundo año, en todas las clases observadas persistió el mismo estilo didáctico, la docente cumple con un ritual que consiste en el saludo, designa dos alumnos que van a la biblioteca a traer libros de Historia, comienza explicar la primera etapa de la Revolución francesa, finalizado el tema, formula preguntas a la clase, los alumnos deben buscar las respuestas en los libros de texto, finalizada esta tarea, se realiza el control en forma oral; finalmente los alumnos tienen como tarea para la casa memorizar las respuestas.

Realmente la concordancia es perfecta, en este caso, la planificación se limita a presentar temas y el desarrollo de la clase de la misma manera: enunciar temas de índole político, es el enciclopedismo en vigencia.

La docente aclara que es única manera que los alumnos estudien Historia

El Profesor José Luis, ejerce en u primer año, se han observado clases, en que se desarrolló el tema Historia, concepto, alcances y ciencias auxiliares (como constaba en la panificación, para un primer año de una ENS -escuela de nivel secundario-). (Es una escuela periférica de un barrio carenciado, con alumnos con problemas de droga, carencias familiares y la infraestructura del establecimiento tiene falencias, específicamente por los robos que sufre).

El profesor escribe el título en el pizarrón Historia, va preguntando a los alumnos, el significado de ese término, la mayoría no responde. El profesor dicta una definición "Es una ciencia que estudia el pasado del hombre" luego escribe en el pizarrón Ciencias Auxiliares, realiza una explicación. Dicta una definición, que son las ciencias que ayudan a la Historia a estudiar el pasado, e inmediatamente les indica que este cuerpo de conocimientos forma parte de la evaluación, da como tarea buscar para la próxima clase que "significan cada una de las ciencias". 
En las siguientes clases, el docente, se limita a llamar a los alumnos requiriéndoles qué respuestas tenían en las carpetas y colocar nota.

De acuerdo con las entrevistas, la planificación y la clase existe concordancia en las concepciones del docente, al decir de Marrero "es un mediador entre el alumno y el conocimiento" de su propio nivel ${ }^{\mathrm{ix}}$

La profesora, Mercedes, explicó que la planificación sólo la tiene en cuenta para orientarse en los temas que desarrolla, porque trata de explicar todos los temas que establece el diseño curricular. A pesar de ello, la docente a medida que explica el tema Gobierno de Juan M. de Rosas, se explaya en los diferentes enfoques que existen sobre el tema, aporta bibliografía personal y la que traen los alumnos (había solicitado búsqueda de material el día anterior), el nivel sociocultural y económico de los estudiantes le permite este tipo de actividades.

No podemos establecer la concordancia, entre la postura docente y la enseñanza, porque si bien la docente no manifiesta una posición frente a las escuelas históricas trata de presentar, a los alumnos, las diferentes perspectiva que distintos que los autores analizan el tema.

La concordancia con la planificación se corresponde porque en los objetivos enunciados por la docente expresa que los alumnos emitan juicio crítico sobre los temas históricos que generan diferentes posturas ideológicas o políticas.

\section{Conclusión}

En las reuniones de los alumnos/pasantes con el equipo de cátedra y docentes tutores, se socializan los datos y existe un intercambio de opiniones al respecto, con las aclaraciones necesarias para una mejor comprensión de la realidad, sobre todo con los aportes de los docentes que ejercen en el nivel medio. De esta manera se identifica la situación de la enseñanza de la Historia en el nivel medio, señalándose:

1.- En cuanto al enfoque de la disciplina Historia, este permanece fiel a la escuela metódica, la cadena lineal de hechos cronológicos prevalece, por lo tanto, los docentes recitan (explican) los temas, de acuerdo con un texto o con la interpretación de los hechos que hace el/la profesor/a, hasta llegar a dictar un resumen de la "lección" que los alumnos deben memorizar. Esta situación es una constante, que se observa sobre todo en las escuelas de zonas periféricas, alegan razones económicas, porque los alumnos no tienen acceso a los libros de textos. La diferencia, con los establecimientos del micro centro, es que en vez de dictar la narrativa, directamente la seleccionan, generalmente, de un sólo texto. En las entrevistas, los/as docentes fundamentan esta postura, afirmando que "es la única forma que los alumnos "aprenden" Historia". Caso contrario "no se acuerdan nada" al finalizar el año; por lo tanto, apelan a la memoria.

2.- Con respecto a las prácticas docentes continúan siendo tradicionales, porque la tarea de enseñar consiste en la transmisión verbal de contenidos, organizados cronológicamente, lo que presupone la base de un conocimiento científico acabado y verdadero que el estudiante debe respetar y apropiarse de ese saber sin modificarlo ni interpretarlo.

3.- Se observa un desfasaje entre lo planificado y el desarrollo de la clase (curriculum prescriptivo y oculto Stenhouse), que consiste en la transmisión de los hechos históricos con perspectiva metódica, sin una relación concatenada de los mismos (Bourdé, Guy.1992), en que el alumno, no construye el conocimiento, sólo se limita a estudiarlo de memoria, en tanto en las planificaciones obran fundamentos, que 
se trabaja a partir de los resultados de un diagnóstico previo para aplicar el constructivismo. En la realidad del aula, no se ha observado, consideración alguna de los saberes previos y se continua con la directiva "para mañana estudian tal tema", ciertos docentes estiman que esta actitud es apelar al constructivismo, porque parte de los conocimientos previos.

Dos docentes consideran - y se ha observado-, que desarrollan sus clases con un enfoque hermenéutico, sin embargo, no respetan la elaboración de los conocimientos que realizan los alumnos, porque dan una interpretación (la de la cátedra) que los alumnos se deben limitar a estudiarla; es decir, estamos ante la presencia de lo que se denomina la vieja-nueva historia, se traduciría en "estudiar de memoria" (vieja historia) la interpretación de los procesos históricos (nueva historia) que realiza el docente, desde su propia perspectiva.

Al reflexionar, sobre estas acciones, en las entrevistas, los docentes justificaban su desempeño en la formación que habían recibido en el profesorado, que se sentían muy marcados por sus formadores y repetían los modelos con los que habían aprendido.

Al realizar la triangulación de los datos se evidenciaron situaciones contrapuestas como ser: 1.- por un lado la correspondencia total entre las concepciones y las planificaciones con una sucesión precisa y minuciosa de hechos históricos. Además, de la ausencia de conceptos puentes para establecer relaciones con las otras disciplinas; no coincidiendo, en este caso, con la respuesta en que establecen que usan la interdisciplinariedad, pero en las observaciones de clases y las planificaciones no queda de manifiesto la interdisciplinariedad. 2.- La otra situación se caracterizó por un tipo de planificación, que al momento de observar la práctica no hay coincidencia (desfasaje planificación - práctica).

Ante esta situación, las reflexiones de los alumnos-pasantes es significativa, en cuanto a la didáctica de la Historia en el nivel medio:

* La enseñanza de la Historia, del nivel medio, es el relato y estudio de la sucesión cronológica de hechos.

* No hay correlación entre los enfoques de la disciplina (Globalizante y procesual, que dicen aplicar, los docentes) con las teorías de enseñanza/aprendizaje constructivismo-.

* Se explicitan los aspectos sociales, políticos, económicos, culturales como factores determinantes de la realidad histórica, sin establecer las relaciones correspondientes y la búsqueda de la comprensión por parte de los alumnos

* En las autobiografías profesionales y entrevistas a los docentes, se observó el cuestionamiento a la formación que habían recibido en "su carrera docente" con respecto a la realidad con la que se encontraron cuando comenzaron a "dar clases"

\section{La formación del profesor en historia}

En virtud de las explicaciones vertidas en párrafos anteriores, los alumnos pasantes, reconocen su situación como paradójica y conflictiva, al momento de iniciar sus prácticas profesionales; especialmente cuando los docentes, en ejercicio, establecen que sus primeras experiencias en la docencia fue traumática y determinan que las causales de todo su quehacer docente, incluidos los temores iniciales se debe a la formación que recibieron en la universidad. Admiten que la formación docente, no 
es tal; que se sienten inseguros al enfrentarse con los adolescentes, al confeccionar una planificación y evaluar pero, muy seguros en los contenidos disciplinares.

Se continúa el proceso de análisis con la formación docente que ofrece la facultad y el sentir de los futuros profesores en Historia. De acuerdo con la reglamentación vigente, se solicita a los alumnos/pasantes, la elaboración de un diario de itinerancia, que debe comenzar con la autobiografía del estudiante universitario y continuar con "su seguimiento" de la formación que reciben. Además, se logró la debida autorización para realizar un análisis en profundidad de sus propios diarios, todos los estudiantes accedieron a entregar los mismos y colaborar en la investigación. Se trabajó sobre un total de 25 diarios de itinerancia.

En primera instancia se realizó una lectura y categorización de las mismas, proceso enriquecedor que se realizó en el curso, con la presencia de todo el grupo; se pasó luego a aplicar los criterios sobre la naturaleza de los aprendizajes profesionales (Develay, 1994; Porlan y Rivero, 1989 y Pérez Gómez, 1992); modelos centrados en la adquisición; en procesos prácticos y en el análisis de lo académico.

Al analizar sus propios diarios de itinerancia y socializarlos en las clases, detectan que el $100 \%$ de los alumnos/pasantes coinciden en los siguientes puntos: ${ }^{x}$

- Valoran la formación en cuanto a contenidos temáticos de la disciplina, que los hace sentir muy seguros en el manejo de los mismos y les proporciona tranquilidad al momento de enseñarlos; pero, el problema reside en cómo o la forma en que se debe enseñar.

- Cuestionan la formación pedagógica que reciben, porque establecen que poseen conocimiento teóricos al respecto, pero no práctico.

Las siguientes citas, corresponden a los diarios de itinerancia de los estudiantes del quinto nivel del profesorado en Historia que cursan la asignatura Didáctica Específica y Pasantía en Historia, en la universidad. La selección de los párrafos que se transcriben, demuestran el sentir y vivir de los alumnos al momento finalizar su carrera y de iniciar la práctica profesional.

"en lo personal considero que la formación recibida en la facultad desde el aspecto propio de la disciplina historia fue muy bueno y completo. Esto lo puedo afirmar con certeza, debido que a través del cursado de las diferentes materias propias de la disciplina y del aprendizaje fue excelente llegando a cumplir con los objetivos y con mis expectativas, sin embargo, me animo a decir que, en cuanto a esta formación considero que sobre el aspecto del rol docente no considero que tenga una formación completa, ya que pienso y de hecho no estaría en una posición segura de afirmar que en el aspecto didáctico de mi formación sea todo completo o la adecuada"(Extraído del Diario de Itinerancia de José)

"Es cierto que la formación que recibimos en la universidad es buena y muy diferente a la que podríamos obtener en otros lugares, pero la misma sólo se aboca a formar profesionales/especialistas en la disciplina, pero no así como docente; ya que esa formación recién la estoy obteniendo ahora. La afirmación que la formación recibida no colmó mis expectativas, es justamente por eso, porque al día de hoy me considero una 'especialista` o conocedora de la disciplina, pero aun no puedo llegar a afirmar que soy docente, porque todavía no encontré 
la forma, y tampoco en las materias del `área de formación docente` me orientaron a encontrar la manera de bajar mi formación disciplinar al aula y saber adecuarla a los alumnos"(extraído del Diario de itinerancia de Jorgelina)

"En lo que respecta a las materias pedagógicas que eran interdisciplinarias, al no ser específicas de nuestra disciplina; nos abrieron un espacio para el conocimiento del "mundo escolar"; pero tal vez no cumplieron con algunas expectativas que teníamos sobre las mismas, porque fue mucha teoría y poca práctica"... Recién con el cursado de esta cátedra pudimos ponernos en contacto el aspecto "cómo" (sic) enseñar Historia; nos brinda espacios de reflexión constante sobre nuestros presupuestos y los pone en inevitables situaciones de toma de decisiones" (extraído del Diario de itinerancia de María Victoria)

Estas reflexiones, señalan claramente que la formación docente que han recibido, de acuerdo con los modelos propuestos por Develay (1994) está centrada en la adquisición de los modos de razonar de la disciplina; en tanto que autores como Porlan y Rivero (1998), prefieren denominar modelo centrado en los contenidos profesionales (científicos y pedagógicos) vistos desde una perspectiva teórica. De acuerdo con Pérez Gómez (1992) es un modelo academicista tradicional, que pone el acento en el "proceso de transmisión de conocimientos", que concibe al docente como un especialista en los contenidos disciplinares que los tiene que transmitir verbalmente en clase, es enciclopedista, porque existe un modelo docente que se basa en la idea de que cuanto mayor cantidad de conocimiento se enseñe y aprendan los alumnos no es sólo excelencia, sino que es ser buen docente.

El buen docente es quien posee un dominio preciso de la disciplina en el sentido de manejar datos, hechos y fechas, que le permitirán transmitirlos fehacientemente a los alumnos, cuanto mayor conocimiento disciplinar posea un docente, podrá ejercer mejor su práctica profesional, es decir, que el ejercicio de la docencia pasa exclusivamente por el conocimiento de "la materia" que conlleva de manera inherente saber transmitirlo. Por lo tanto la concepción de enseñar, es transmitir a otros (alumnos) para que aprehendan los conocimientos, desarrollados de acuerdo con fundamentos científicos y lógicos preestablecidos y sólo deben memorizarlos. El dominio de la disciplina es la condición necesaria y suficiente en la formación docente, porque ésta determina su desempeño como buen profesor. Así, la perspectiva academicista no otorga valor relevante a la formación en el área pedagógica, porque al dominar a disciplina no se necesita ninguna otra formación para enseñarla.

- Admiten que se sienten "muy marcados" por las clases magistrales de los docentes en las aulas universitarias. Además, reconocen que admiran ese "modelo de enseñanza" y estiman incuestionable poseer y manejar gran cantidad de contenidos disciplinares.

"El transcurso de estos años en la facultad fue de aprendizaje muy diverso. Si solamente considero lo estrictamente académico puedo decir que recibí una formación tanto en la especialidad de Historia y otra en la pedagógica. Si bien cada una de ellas pueden tener sus críticas, también de esas mismas críticas que fueron surgiendo en el transcurso de mi vida universitaria forman parte de mi formación. Siguiendo el concepto de formación de Ferry fue un proceso de transformación en mi para llegar a desempeñarme como futura docente. En esta transformación que se fue dando considero que todavía hay más para aprender y creo que a lo 
largo de mi vida voy a seguir aprendiendo, si bien anteriormente resalte (sic) la educación formal, también la vida en la universidad me marcó para ser una docente excelente." (Extraído del Diario de Itinerancia de Adriana)

Ante esta situación de quejas por un lado y de ponderación positiva por el otro, se inserta la formación docente; por ello la cátedra Didáctica Específica en Historia finaliza solicitando a los estudiantes propuestas de cambios pertinentes y necesarios para reencaminar su formación.

En las clases, se continúan con las reflexiones de los estudiantes, con respecto a su formación, y los debates se plantean en torno a lo disciplinar y al campo pedagógico concluyendo lo siguiente:

$\checkmark$ Cuestionan la acotada formación pedagógica que reciben,

$\checkmark$ Valoran la buena formación en lo disciplinar (en cuanto a contenidos temáticos)

$\checkmark$ Carecen de conocimiento en cuanto a los fundamentos epistemológicos de la disciplina, como ciencia y desconocen la metodología de trabajo y los aportes de las corrientes historiográficas en vigencia

$\checkmark$ Reconocen que salen bien preparados disciplinarmente; no así como docentes.

$\checkmark$ Admiten que se sienten "muy marcados" por las clases magistrales de los docentes en las aulas universitarias. Les resulta difícil desprenderse de ese estilo de enseñanza y al momento de desempeñar el rol docente, se convierten en docentes ejecutivos (Fenstegmacher y Soltis 1999) pero todos pretender ser un docente "liberador". Por lo tanto las estrategias de enseñanza continúan siendo tradicionales con una concordancia total con el enfoque de la disciplina, es decir permanencia en la escuela metódica (Historia) y el modelo conductista, en que el docente es fiel "repetidor" de hechos históricos y los alumnos los deben memorizar.

$\checkmark$ Reconocen que la tarea de ser docentes queda en sus manos, que la universidad, de forma urgente, debe replantearse la formación que brinda en el profesorado en Historia; como así también debe existir un acompañamiento de cambio por parte del cuerpo docente.

Finalmente los alumnos/as-pasantes, a través de los años estiman necesario tener en cuenta las siguientes propuestas:

$\checkmark \quad$ En el desarrollo de las clases en la universidad, aplicar una didáctica hermenéutica y crítica en todas las cátedras, para tomar como modelos en sus prácticas docentes.

$\checkmark$ Implementar la interdisciplinariedad, abordando los acontecimientos históricos desde una perspectiva múltiple y reticular

$\checkmark$ Conocer los criterios de selección de los contenidos (que realizan los docentes universitarios) para determinar, desde qué enfoque disciplinar enseñan (y se puede enseñar). Es uno de los puntos críticos, porque les resulta difícil seleccionar contenidos, pretenden enseñar "todo" lo que aprendieron en el profesorado (formación academicista) 


\section{Conclusión}

En virtud de lo expuesto, en líneas generales, se caracterizó a los profesores de Historia y la didáctica de la disciplina, como:

A. un docente sumergido en la tendencia de la didáctica tradicional, que aún no implementan la enseñanza de la disciplina a través de técnicas procedimentales y, que se puede "usar" la memoria comprensiva.

B. un docente que no establece la correlación entre los fundamentos epistemológicos y enfoques historiográficos de la disciplina con las teorías de enseñanza/aprendizaje Por lo general determinan, desde lo histórico, una caracterización de los aspectos sociales, políticos, económicos, culturales como factores determinantes de una realidad histórica, sin establecer las relaciones correspondientes entre cada aspecto que, los alumnos deben estudiarlos "de memoria", sin comprender la correlación

El proceso de análisis, en el trabajo de investigación realizado, a medida que se profundiza y se extiende a un número mayor de profesores, se concluye en que:

a) Existe una cierta coherencia entre las creencias, concepciones o teorías del profesor y su práctica educativa. La toma de decisiones del docente referida a la enseñanza, ya sea a la hora de planificar, o en la interacción del aula, o en la evaluación posterior, está determinada por sus concepciones sobre el conocimiento (científico), la enseñanza, el aprendizaje y la función social de la escuela ${ }^{\mathrm{xi}}$. Estas concepciones, supuestos, teorías las ha ido construyendo a lo largo de su historia educativa, ya sea desde el rol de alumno o de docente, a través de la experiencia y la socialización profesional, constituyendo su conocimiento profesional (Porlán, R. y Rivero, A., 1998), que orienta y da un estilo a las prácticas pedagógicas que realiza.

b) Se coincide con las observaciones y conclusiones de los estudios sobre el pensamiento del profesor respecto de la ciencia y las corrientes historiográficas, que señalan un modelo dominante o hegemónico en la enseñanza de la Historia, cuya base epistemológica proviene del positivismo (corriente metódica) y el empirismo lógico. Su base psicológica remite a la concepción de aprendizaje como un proceso lineal, descontextualizado, de acumulación de información y su correspondencia con la concepción de la disciplina como narración cronológica "lineal" de hechos históricos; aunque algunos docentes, como lo establecimos anteriormente, determinan una caracterización de los aspectos sociales, políticos, económicos, culturales como factores determinantes de una realidad histórica, sin establecer las relaciones correspondientes y la búsqueda de la comprensión por parte de los alumnos

c) Desde el punto de vista Didáctico, esto implica prácticas transmisoras del profesorado, orientadas a la memorización y la obtención de respuestas correctas por parte de los alumnos. En este sentido, se considera que dicho modelo es el generalizado en la enseñanza de los contenidos disciplinares de otros campos científicos, debido a que constituye la manera en que los formadores de docentes hemos sido formados, es decir, "es la única forma como sabemos hacerlo" (Porlán, 1997:140).

d) En relación con lo anterior, se supone que si bien los estudios relevados se refieren a profesores de nivel medio en ejercicio o en formación, los 
resultados serían coincidentes en el caso de formadores de los docentes universitarios, por el hecho de que reconocen que son tomados como modelo.

Estos supuestos llevan a plantear el problema de la formación docente en relación con el cambio educativo, de cara a los paradigmas didácticos que intentan superar el reduccionismo inherente a la enseñanza como transmisión de saberes. Por otro lado, tales paradigmas se apoyan en las explicaciones de las teorías de aprendizaje cognitivo/constructivistas, que han demostrado que el aprendizaje en sentido estricto supone la participación activa del sujeto, ya que es él quien construye sus propios conocimientos, en interacción con los demás y con el objeto de conocimiento, elaborando y reelaborando las nuevas informaciones en función de sus aprendizajes precedentes.

Desde estos paradigmas didácticos actuales, la enseñanza como facilitadora del aprendizaje en términos de construcción, se basa también en una concepción del conocimiento científico y de sus procesos de elaboración e interpretación diferente a la concepción epistemológica de la enseñanza tradicional. Desde esta perspectiva, las concepciones sobre la naturaleza de la ciencia, las corrientes historiográficas y del método científico de los profesores universitarios, influirían en la toma de decisiones sobre la enseñanza y el aprendizaje en las materias y cátedras donde se forman los futuros docentes de Nivel Medio y Nivel Superior.

De acuerdo con el presente planteo, se considera que uno de los problemas centrales de la formación docente universitaria es que, el modelo de enseñanza que vivencian los estudiantes estaría contribuyendo a la permanencia de una visión determinada de la ciencia, que no se adecuaría a las formulaciones actuales de la epistemología ni de las corrientes historiográficas, como tampoco a los paradigmas didácticos orientados a un protagonismo genuino del alumno en la adquisición del conocimiento.

Una cuestión que se debe tener presente, al momento de analizar la formación docente, es una situación que en los últimos años, se viene detectando: los estudiantes que inician la carrera del Profesorado en Historia, no tienen conciencia del significado de ser docentes, la mayoría establece que se inscribió en la carrera porque le gustaba Historia, cuando comienzan a cursar Didáctica Específica (en el cuarto nivel), se dan cuenta que su profesión es la docencia, en este momento se cuestionan y piensan en la transposición didáctica; esa gran cantidad de contenidos disciplinares, que ponderan haber recibido, ahora deben seleccionar para enseñar en el nivel medio. El imaginario del profesor ideal, el que puede lograr que los adolescentes "estudien" Historia, es tema de continuos debates.

\section{Referencias}

AA.VV. (1999) Desarrollo profesional del docente: Política, investigación y práctica. Madrid. Akal.

Braudel, F. (1999) La historia y las ciencias sociales. Madrid: Alianza.

Bourde, G. (1992) Las escuelas históricas. Madrid. Akal.

Carr y Kemmis, (19988) Teoría crítica de la enseñanza .Madrid: Martínez Roca.

Eggen, P. y Kauchak, D. (1999) Estrategias docentes. Enseñanza de contenidos curriculares y desarrollo de habilidades de pensamiento. Brasil: F.C.E.

Fenstermacher, G. y Solti, J. (1999) Enfoques de la enseñanza. Buenos Aires: Amorrortu. 
Fontana, J. (2001) La historia de los hombres. Barcelona: Crítica.

Joyce, B. y Weil, M. (2000) Modelos de enseñanza. España: Gedisa.

Koselleck, R. (2001). Los estratos del tiempo: estudio sobre la historia. Barcelona: Paidós.

Lopez Ruiz, J. I. (1999) Conocimiento docente y práctica educativa. El cambio hacia una enseñanza centrada en el aprendizaje Málaga: Aljibe.

Marrero, J. (1993) Las teorías implícitas del profesorado: vínculo entre la cultura y la práctica de la enseñanza. En Rodrigo, M. J. Las teorías implícitas. pp.24 Madrid: Visor.

Moradiellos, E. (2001) Las caras de Clío. Siglo XXI.

Pérez Gómez, A. (1989) Paradigmas contemporáneos de investigación didáctica. En Gimeno Sacristán, J. y Pérez Gómez, A. (Comp.) La enseñanza: su teoría y su práctica. Madrid: Akal.

Pérez Gómez, A. (1992) Enseñanza para la comprensión. En Gimeno Sacristán, J. y Pérez Gómez, A. Comprender y transformar la enseñanza. Madrid: Morata.

Porlan, R. y Rivero, A. (1998) El conocimiento de los profesores. Una propuesta formativa en el área de ciencias. España: Díada.

Pozo, J. I. y Perez Echeverria, M. del P. (coord) (2009) Psicología del aprendizaje universitario: la formación en competencias. Madrid: Morata.

Rodrigo, M. J., Rodriguez, A. y Marrero, J. (1993) Las teorías implícitas. Una aproximación al conocimiento cotidiano. España: Visor.

Shulman, L. (1990) Paradigmas y programas de investigación en el estudio de la enseñanza: una perspectiva contemporánea. En Witrock, M. La investigación de la enseñanza. Enfoques teorías y métodos. (cap.lpp.9 - 95) T.I. Barcelona: Paidós.

Schön, D. A. (1992) La formación de profesionales reflexivos. Buenos Aires: Paidós.

Stenhouse, L. (2010) Investigación de desarrollo del currículo. Madrid: Morata.

Rockwell, E. (2000) La escuela cotidiana. España: Visor.

Witrock, M. (1989) La investigación de la enseñanza. Enfoques teorías y métodos T.I y T II.

\footnotetext{
i Para el presente trabajo se han tenido en cuenta las observaciones realizadas durante el periodo 2013. Los alumnos pasantes, cuentan con las conclusiones de los compañeros de años anteriores. Precisamente desde el año 2006, en que se han sistematizado las técnicas de análisis aplicadas.

ii Las entrevistas se realizan a todos los profesores en Historia de los establecimientos secundarios, totalizando 32 docentes y, el análisis de las planificaciones se remiten sólo de los cursos que observan, dos por escuela es decir 12 en total.

iii López Ruiz, J. I. (1999) Conocimiento docente y práctica educativa. Málaga: Aljibe. p. 80

iv Porlan y Martín, citado por López Ruiz, J. I. Ob.cit.
} 


\footnotetext{
${ }^{\vee}$ Moradiellos, E. (2001) Las caras de Clío. siglo XXI p. 60

vi Koselleck, R. (2001) Los estratos del tiempo: estudio sobre la historia. Barcelona: Paidós

vii Marrero, J. (1993) Las teorías implícitas del profesorado: vínculo entre la cultura y la práctica de la enseñanza. En Rodrigo, M. J. Las teorías implícitas. Una aproximación al conocimiento cotidiano. Madrid: Visor. p. 243.

viii'Witrock, M. (1989) La investigación de la enseñanza T .II. p. .364

${ }^{i x}$ Marrero, J. (1993) Las teorías implícitas del profesorado: vínculo entre la cultura y la práctica de la enseñanza. Ob.cit. p.24.

× El grupo curso acordó las narraciones que debían figurar, como representativa del sentir de todos, en el informe de investigación

${ }^{x i}$ La concepción sobre la función social de la escuela tiene una base fuertemente ideológica referida a las relaciones entre Estado, Sociedad y Educación que no se abordarán en este estudio, pero que sin duda se relacionan con las concepciones sobre ciencia, enseñanza y aprendizaje, y estarían operando como marco general cultural, social e históricamente compartido. Prof. María Teresa Alcalá 2010.
} 